\title{
Comments on "Publisher's Note" on papers on individual external dose monitoring of all citizens of Date City
}

\author{
Yoh Tanimoto \\ Dipartimento di Matematica, Università di Roma "Tor Vergata" \\ email: hoyt@mat.uniroma2.it \\ Yutaka Hamaoka \\ Faculty of Business and Commerce, Keio University \\ email: hamaoka@fbc.keio.ac.jp \\ Kyo Kageura \\ Graduate School of Education, University of Tokyo \\ email: kyo@p.u-tokyo.ac.jp \\ Shin-ichi Kurokawa
}

The High Energy Accelerator Research Organization (KEK), Tsukuba

email: shin-ichi.kurokawa@kek.jp

Jun Makino

Department of Planetology, Graduate School of Science, Kobe University

email: makino@mail.jmlab.jp

Masaki Oshikawa

Institute for Solid State Physics, University of Tokyo

email: oshikawa@issp.u-tokyo.ac.jp

Dear Sir,

We point out that the recently published Publisher's Note [1] concerning two retractions in JRP and the retraction notices $[2,3]$ contain various incorrect statements, misrepresentations of facts and omission of important events.

\section{Retraction notices}

(1) The summary of the report by Date City Citizen's Exposure Data Provision Investigation Committee is incorrect: the retraction notices write "which finds that some subjects within the study did not consent to their data being used for research, and it is unclear whether the unconsented data was provided to the author". In fact, in the report $[4$, P.12, (4)(1)Conclusion], it is clearly stated that "It was inappropriate for the City that 
it did not verify that the data on external doses contained participants who had not given consent and that it provided the researchers with the data without deleting the data of those who had not given consent" (translated by us). On the other hand, the investigation report has nothing to say on the question of whether the data were used for research, because the scope of the investigation committee [4, P.3, (3) Scope of the investigation] was examination of administrative procedures and handling of personal information by Date City. Thus its mandate did not include examination of the contents of the papers. Therefore, the retraction notices are doubly incorrect in that it fails to acknowledge the fact that was confirmed in the report and it misrepresents the contents of the report.

(2) In [2, 3] URL https://www.city.fukushima-date.lg.jp/soshiki/3/41833.html is linked to a summary of actions of Date City, which contains neither the investigation report itself nor a link to it https://www.city.fukushima-date. $1 \mathrm{~g}$.jp/uploaded/ attachment/43948.pdf. This suggests that the editorial office of JRP has neither read the report nor consulted anyone who could read it.

(3) $[2,3]$ call the source of the data "a third party". This is inappropriate, because the source is Date City, M. Miyasaki was a policy adviser of Date City, and the whole research has been conducted on request by the Date City mayor, as stated in the research protocol submitted to Fukushima Medical University.

(4) $[2,3]$ state that "the authors of the above referenced article confirmed that the research reported in the article adhered to the Ethical Policy of IOP Publishing and the Society for Radiological Protection." While it might be true that the authors claimed it, it is clear that the authors had not properly disclosed their conflicts of interest: M. Miyasaki was a policy adviser of Date City, and analysis of data and writing papers in some international journal to publicize the results has been conducted by the request of then Date City mayor.

(5) [2] states that "Based on the investigation report it has also been found that there is an error in table 1 of this article. The figure relating to glass badge holders in $20143 \mathrm{Q}$ is incorrect and should be close to $\mathrm{N}=12912$." What the investigation report of Date City actually shows is the numbers of participants who held grass badge continuously for one year (from July to June) between July 2012 and June 2018 ([2] first cited the number 12011 that corresponds to the period from July 2015 to June 2016, and then has corrected it on 6 November 2020 to 12912, the number for the period from July 2014 to June 2015). It is unusual that that the journal states only a mistake in number, without details of how this was found while the authors had deleted the data, and without discussing the consequences of this error. If the authors did not have the correct data of $20143 \mathrm{Q}$, then [5, Figure 4] must be incorrect and the coefficient $c$ must be recalculated. Furthermore, [6, Figure 5] includes the same period, and must be affected as well.

(6) [2] states that "authors were not aware of this mistake in advance of publication of the article". In fact, in one message (made public by a FOI request, see [7] for an extract of the email) from Date City on 3 August 2015, Miyazaki was informed that the glass badge data from 2014 Q2 to 2015 Q1 would not be provided. Furthermore, another document 
made public by a FOI request to Date City [8, P.2, upper right figure], created most probably by the authors, reads "GB period 2013/10-12: The 9th airborne monitoring $(2014 / 11 / 7)$ ". That is, it is clearly stated that they compared the glass data in 2013 and the airborne monitoring data in 2014. This strongly suggests that the authors knew that the glass badge data were not of the period 2014 3Q, and hence the above statement given in [2] is incorrect.

(7) It was our Letter 3 [9] that found the mistake in the data of 2014 3Q and pointed out that the correct number should be about 14500 (this Letter and all other Letters were provisionally accepted (Letter 1 was accepted formally) and we were waiting for the reply by the authors): FMU has confirmed that Miyazaki came to know this error through an email from Ms. Shiraishi of OurPlanet-TV, while OurPlanet-TV was informed of it by a "team of researchers including Prof. Kurokawa" [10]. Yet the retraction notice [2] mentions this error without any reference to our Letter. Furthermore, [2] does not cite any source nor justification, and it is entirely unclear how JRP obtained the number "N = 12912" and judged that it is not "ethically inappropriate", while withdrawing our Letters.

\section{Publisher's Note}

(8) [1] discusses no scientific errors in the original papers, although JRP has published a Expression of Concern on [6] on "a methodological miscalculation underpinning the reported results", the authors have admitted their miscalculation and the investigations by their universities have confirmed it. JRP, therefore, retracted a paper only on an ethical basis, and did not mention that EOC highlighted that the article contained a methodological miscalculation underpinning the reported results. The miscalculation may affect the main conclusions of the article.

(9) Our Letters did not use unconsented data. It makes no sense to state that JPR could not verify it, because it made no reasonable effort to verify it: JRP has asked no question to us about how we obtained our conclusions, and the only thing it observes is that our Letters cites the original figures and formulas. But citing retracted papers should not be confused with using the original unconsented data.

(10) It is remarkable that the University of Tokyo has refused to "enter into any correspondence with IOP Publishing on the matter or respond to any questions asked". The journals rely on academic institutions for investigations into possible scientific misconducts. Lack of cooperation from the institutions might deserve to be highlighted.

(11) As in retraction notices, it is inappropriate to call Date City a "third party."

Timeline In the timeline shown in [1], important events are missing or misrepresented. These include the following.

(12) (27 November 2018) The Japanese national policy regarding the medical research ethics, whose article on research data has been effective since 1 October 2015 (hence before the 
ethical approval of the original studies) [11], requires that the dataset be preserved as long as possible, instead of being discarded.

(13) (12 December 2018) JRP decided not to publish Letter 1, as the authors would write a corrigendum. This decision would be reconsidered on 29 January 2019 and JRP promised that it would publish Letter 1 even if the original papers would be retracted.

(14) (7 January 2019) The Date City resident mentioned here has informed the IOP publishing regarding not only potentially ethical misconduct but also scientific misconduct.

(15) (8 January 2019) Hayano posted a note on twitter [12] admitting the miscalculation in [6]. However, later he withdrew this explanation [13].

(16) (11 January 2019) The investigation by Date City had started on 4 February, therefore, it was not "underway" on 11 January. In addition, JRP had decided not to publish Letter 1 on 12 December 2018, and the decision was reversed only on 29 January 2019. It was impossible for JRP to make Letter 1 on hold on 11 January 2019.

(17) (19 July 2019) The investigations of the University of Tokyo (UT) and Fukushima Medical University (FMU) concluded. Hayano changed the account for the mistakes in [6] on twitter [13].

(18) (15 Aug 2019) This entry misrepresents the investigation reports:

- FMU admitted that unconsented data were used. It only stated that the authors could not be held responsible for it. UT did not investigate the ethical aspects.

- FMU report is wrong about the lifetime doses. It is clear that the lifetime doses were underestimated [14].

(19) (4 March 2020) Letter 3 is authored by all of us.

(20) (17 March 2020) The investigation of Date City concluded. It should be noted that this was communicated to JRP by Miyazaki only in June, even though "IOP Publishing had routinely requested updates on the progress of the investigations" and the authors "were responsive and helpful" [1].

(21) (18 March 2020) Letter 1 was provisionally accepted.

(22) (23 March 2020) Letter 1 was formally accepted and JRP sent a message to Kurokawa saying "We will contact you again soon when proofs of your article are ready for final approval." This clearly shows that Letter 1 was not on hold.

(23) (7 April 2020) By this date, Letter 2, 3, and 4 had been provisionally accepted. Also we had been informed that JRP was waiting for the replies of the original authors. We were never informed by JRP that the Letters were on hold because of the Investigation of the Date City committee was underway.

Actually, the Inverstigation of the Date City committee has concluded on 17 March 2020, hence it is impossible for JRP to place Letter 4 on hold. 
Lastly, we are of the opinion that the reasoning in [1] "Since IOP Publishing was not provided specific details of the ethically inappropriate data, it was not felt possible to independently verify with certainty whether or not the four Letters were free of the use of the ethically inappropriate data." is rather irresponsible and cannot be a basis for not publishing scientifically sound criticisms. If the IOP Publishing is unaware of "specific details", it should seek for necessary information before making the important decision such as refusing publication of Letters which are already accepted. We would be happy to help if the IOP Publishing wishes (as it should) to contact relevant authorities in Japan. The consequence of its logic must be that the part of the Letters which are "free of the use of the ethically inappropriate data" should be published. We believe that entire Letters are free of such problems, and the onus is on the publisher to prove with "specific details" if JRP insists there is an ethical problem with our Letters.

To summarize, it is preposterous that an editorial which is supposed to conclude a case of important retractions contains such many errors, misinformation and omission of important facts. Some of them show that JRP and IOP Publishing have not investigated the case properly. We hope that JRP fulfills its mission as a scientific journal.

\section{Acknowledgements}

We thank Ms. Akemi Shima for providing us with the public documents obtained through her Freedom Of Information requests.

We thank the KAGAKU Editorial Office (Iwanami Shoten, Publishers) for opportunities to discuss this work.

\section{Conflicts of interest}

None of the authors have any conflicts of interest or financial ties to disclose.

\section{References}

[1] Publisher's note on: 'individual external dose monitoring of all citizens of date city by passive dosimeter 5 to 51 months after the fukushima NPP accident (series): I. comparison of individual dose with ambient dose rate monitored by aircraft surveys' (2017 j. radiol. prot. 371 ) and 'individual external dose monitoring of all citizens of date city by passive dosimeter 5 to 51 months after the fukushima NPP accident (series): II. prediction of lifetime additional effective dose and evaluating the effect of decontamination on individual dose' (2017 j. radiol. prot. 37 623). Journal of Radiological Protection, 40(3):E19-E23, sep 2020. https://doi.org/10. 1088/1361-6498/aba42a.

[2] Retraction: Individual external dose monitoring of all citizens of date city by passive dosimeter 5 to 51 months after the fukushima NPP accident (series): 1. comparison of individual dose with ambient dose rate monitored by aircraft surveys $(2017 \mathrm{j}$. radiol. prot. $37 \mathrm{1})$. Journal of Radiological Protection, 40(3):908-909, jul 2020. https://doi.org/10.1088/1361-6498/ ab9ffo.

[3] Retraction: Individual external dose monitoring of all citizens of date city by passive dosimeter 5 to 51 months after the fukushima NPP accident (series): II. prediction of lifetime additional 
effective dose and evaluating the effect of decontamination on individual dose (2017 j. radiol. prot. 37 623). Journal of Radiological Protection, 40(3):906-907, jul 2020. https://doi.org/ 10.1088/1361-6498/ab9ff1.

[4] Date city citizen's exposure data provision investigation committee report. mar 2020. https: //www.city.fukushima-date.lg.jp/uploaded/attachment/43948.pdf.

[5] Makoto Miyazaki and Ryugo Hayano. Individual external dose monitoring of all citizens of Date City by passive dosimeter 5 to 51 months after the Fukushima NPP accident (series): 1 . Comparison of individual dose with ambient dose rate monitored by aircraft surveys. Journal of Radiological Protection, 37(1):1-12, 2016. https://doi.org/10.1088/1361-6498/37/1/1.

[6] Makoto Miyazaki and Ryugo Hayano. Individual external dose monitoring of all citizens of Date City by passive dosimeter 5 to 51 months after the Fukushima NPP accident (series): II. Prediction of lifetime additional effective dose and evaluating the effect of decontamination on individual dose. Journal of Radiological Protection, 37(3):623-634, 2017. https://doi.org/ 10.1088/1361-6498/aa6094.

[7] Shin-ichi Kurokawa. Further issues with the papers on big radation dose data analysis. Kagaku, 5(90):e001, 5 2020. https://www.iwanami.co.jp/kagaku/eKagaku_202003_Kurokawa.pdf.

[8] Date city. The 9th airbone monitoring data. August 2015.

[9] Shin-ichi Kurokawa, Masaki Oshikawa, Yutaka Hamaoka, Kyo Kageura, Jun Makino, and Yoh Tanimoto. Further comments on "Individual external dose monitoring of all citizens of Date City by passive dosimeter 5 to 51 months after the Fukushima NPP accident (series): 1." : Inconsistencies in Table 12014 Q3 and Figure 4f. https://arxiv.org/abs/2003.05403.

[10] OurPlanet-TV. A news article on 11 october 2020. http://www. ourplanet-tv.org/?q=node/ 2524.

[11] The Ministry of Education, Culture, Sports, Science and Technology. Ethical guidelines for medical and health research involving human subjects. https://www.lifescience.mext.go . jp/files/pdf/n2181_01.pdf.

[12] Ryugo Hayano. https://twitter.com/hayano/status/1082488374043103232.

[13] Ryugo Hayano. https://twitter.com/hayano/status/1152129151786049542.

[14] Shin-ichi Kurokawa and Yoh Tanimoto. Comments on the investigation reports by Fukushima Medical University and the University of Tokyo on allegations regarding papers on radiation dose estimates in Date City. KAGAKU, 89(8):e0001-596, 2019. https://www . iwanami.co. jp/kagaku/eKagaku_201908_Kurokawa\&Tanimoto.pdf. 